\section{Controversias en el manejo de la fractura de cadera en el adulto mayor. Encuesta nacional a Traumatólogos especialistas en cirugía de cadera}

TOMÁS ZAMORA ${ }^{1}$, IANIV KLABER ${ }^{1}$, FRANCISCO BENGOA ${ }^{1}$, EDUARDO BOTELLO ${ }^{1}$, DANIEL SCHWEITZER ${ }^{1}$, PEDRO AMENÁBAR ${ }^{2}$

\section{Management of hip fractures in the elderly. A national survey among Chilean orthopaedic surgeons}

Background: As the population ages, patients exposed to osteoporotic fractures increase, especially hip fracture, which is the most severe and costly. Aim: To characterize surgical practices in the management of hip fractures in older patients through a nationwide survey of specialized hip surgeons dedicated to the care of these patients. Material and Methods: A survey composed of 32 questions was formulated, including demographic factors, preoperative evaluation, definitive treatment, and postoperative management. It was sent to 140 specialists. 84 of them replied (61\%), and 71 answers were included. Results: Eighty six percent of respondents agreed that orthogeriatric management is fundamental in the outcome of these patients, but only $73 \%$ had the collaboration of an internist or a geriatrician. Although $97 \%$ considered 72 hours or less the ideal time to perform surgery, only $52 \%$ of the respondents declared performing surgery within that timeframe, with differences between private and public system. Regarding surgical treatment, 94-98\% of femoral neck fractures are treated with an arthroplasty and 98-99\% of per-subtrochanteric fractures are treated with internal fixation and osteosynthesis. Osteoporosis treatment is only carried out by $51 \%$ of the respondents and with significant variation. Conclusions: This survey shows that there is agreement in surgical practice between specialists treating these patients, but clear differences in preoperative optimization, treatment timeframe, and post fracture medical treatment.

(Rev Med Chile 2019; 147: 199-205)

Key words: Geriatrics; Hip Fracture; Orthopedics; Osteoporotic fractures.
'Departamento de Ortopedia y Traumatología. Escuela de Medicina, Pontificia Universidad Católica de Chile. Santiago, Chile. ${ }^{2}$ Departamento de Ortopedia y Traumatología. Clínica Alemana de Santiago. Chile.

Trabajo no recibió financiamiento. Los autores declaran no tener conflictos de interés.

Recibido el 6 de octubre de 2018, aceptado el 7 de marzo de 2019.

Correspondencia a: Dr. Tomás Zamora H. Departamento de Ortopedia y Traumatología.

Escuela de Medicina, Pontificia Universidad Católica de Chile. Diagonal Paraguay 362. Santiago, Chile.

tzamora@med.puc.cl
A medida que la población continúa envejeciendo, las fracturas por fragilidad representan una situación crítica y creciente a nivel mundial ${ }^{1,2}$. Dentro de ellas, la fractura de cadera (FC) es la más grave, estando asociada a una morbi-mortalidad elevada ${ }^{3}$ y un costo económico importante ${ }^{4}$.

A nivel internacional, la FC tiene una incidencia entre 414-957 casos por cada $100.000 \mathrm{hab} / \mathrm{año}^{5}$, con más de 200.000 casos al año en los EEUU, cifra que se espera aumente al doble para el año $2040^{6,7}$. En Chile, 9,5\% de los institucionalizados mayores de 60 años ha sufrido una $\mathrm{FC}^{8}$.

Existe amplia evidencia sobre los beneficios de un manejo activo, precoz y multidisciplinario en el tratamiento de los pacientes con fractura de cadera ${ }^{1,2}$. Esto incluye una estabilización de comorbilidades, cirugía pronta y una rehabilitación adecuada, para así alcanzar los mejores resultados. Sin embargo, muchos puntos como la 
urgencia del manejo quirúrgico, el tipo de cirugía a realizar y el manejo metabólico posterior, aún son temas de debate. Más aún, reportes locales que muestran tiempos de latencia hasta la cirugía de 19 días promedio 9 , nos hablan de la falta de guías que regulen el manejo y, por lo tanto, de la falta de un diagnóstico global desde dónde poder realizar intervenciones.

Es así, como nuestro objetivo es caracterizar las conductas de manejo de la FC en el adulto mayor $(\mathrm{AM})$, realizando una encuesta a nivel nacional a los traumatólogos dedicados al manejo de este tipo de pacientes. De la misma forma, comparar prácticas según las características laborales de los encuestados.

\section{Materiales y Métodos}

Se solicitó la aprobación del Comité de Ética Institucional para realizar este estudio.

Para el desarrollo de la encuesta, comenzamos con una aproximación de muestreo hasta la redundancia, en la cual un grupo de traumatólogos especialistas en cirugía de cadera, aportaban temas considerados relevantes hasta saturarlos. Luego de esto, se seleccionaron los temas más importantes para formular las preguntas.

Se realizó un testeo inicial de la encuesta en un grupo de 5 especialistas, para verificar la validez aparente (que el cuestionario como un todo reflejara adecuadamente la pregunta sobre el manejo de la FC en el AM) y de contenido (que cada pregunta individual investigara de forma adecuada el objetivo de este estudio), comentando además sobre la claridad y comprensión de las preguntas. Posterior a esto, la encuesta fue completada por un grupo de 10 participantes para corroborar la validez de contenido y establecer el acuerdo intraobservador del instrumento, tras lo cual quedó consolidada la encuesta definitiva.

El cuestionario final se compuso de 32 preguntas (de alternativas, abiertas cortas, escala tipo Likert y basadas en casos) las cuales fueron divididas en 4 categorías: datos demográficos del encuestado; evaluación preoperatoria; manejo definitivo, incluyendo tipo de cirugía a utilizar; y manejo postoperatorio, con énfasis en rehabilitación y manejo metabólico.

La encuesta fue administrada por correo electrónico utilizando el sistema SurveyMonkey (www.surveymonkey.com) y fue enviada a todos los miembros del Comité de Cadera de la Sociedad Chilena de Ortopedia y Traumatología (SCHOT). Además de esto, se les envió la encuesta a los asistentes Simposio Sudamericano de Actualización en Cirugía de Cadera 2017, realizado por la Pontificia Universidad Católica de Chile. La encuesta fue reenviada en 3 oportunidades de forma personalizada. Las respuestas fueron manejadas de forma anónima, sin ningún estimulo económico para completarla, solicitando a cada encuestado solo responderla una vez. Se excluyeron respuestas incompletas, aquellas completadas por cirujanos que se desempeñen en el extranjero o que declararan tratar menos de $5 \mathrm{FC}$ al año y médicos en etapa de formación.

Se realizó una estimación del tamaño muestral y el mínimo de respuestas necesarias. Para una población estimada de 140 especialistas, un nivel de confianza de $95 \%$ y un margen de $\pm 10 \%$, el número de respuestas necesarias es de 58 cuestionarios (tasa de respuesta de $41 \%$ ).

El análisis estadístico se realizó con el programa SPSS versión 21 (SPSS, Chicago, IL, EEUU). Las variables categóricas fueron comparadas con el test de Chi cuadrado o Fisher según correspondiera. Se compararon respuestas de forma dicotómica según lugar de trabajo del encuestado (establecimiento público/privado o institución académica/ no académica). Por tratarse de múltiples comparaciones con la misma variable dependiente se realizó una corrección por Bonferroni para reducir el riesgo de error tipo 1, obteniendo un alfa corregido $(\mathrm{p}<0,0125)$ para ser considerado como una diferencia significativa.

\section{Resultados}

De un total de 140 especialistas, obtuvimos 84 respuestas $(61 \%)$. De estas, 71 fueron incluidas. Las características de los encuestados están descritas en la Tabla 1.

$\mathrm{Al}$ interrogar sobre el manejo conjunto de los pacientes, $73 \%$ (52/71) declaró que contaba con colaboración regular de un médico internista/ geriatra para la optimización preoperatoria. De la misma forma, $86 \%$ (61/71) se declaró de acuerdo/ muy de acuerdo con que el manejo orto-geriátrico era fundamental en los resultados posterior a una FC. 
Con respecto al tiempo de demora antes del tratamiento quirúrgico en un $\mathrm{AM}$ con una $\mathrm{FC}$, solo el $52 \%$ de los encuestados refirió que el tiempo hasta la cirugía era menos de $72 \mathrm{~h}$ (37/71, Figura 1), sin embargo, 97\% (69/71) consideró este plazo como ideal y $92 \%$ (65/71) se declaró de acuerdo o muy de acuerdo con que el manejo precoz es fundamental para los resultados postoperatorios.

Tabla 1. Datos demográficos de los encuestados

\begin{tabular}{|lr|}
\hline Región & \\
Metropolitana & $44 / 71(62 \%)$ \\
Atacama & $1 / 71(1 \%)$ \\
Valparaíso & $5 / 71 \quad(7 \%)$ \\
O'Higgins & $1 / 71(1 \%)$ \\
Maule & $3 / 71(4 \%)$ \\
Bío-Bío & $8 / 71(11 \%)$ \\
Araucanía & $4 / 71(6 \%)$ \\
Los Lagos & $4 / 71(6 \%)$ \\
Magallanes & $1 / 71(1 \%)$ \\
Tipo de establecimiento & \\
Público & $35 / 71(49 \%)$ \\
Privado & $28 / 71(39 \%)$ \\
Otro (Mutual o FFAA) & $8 / 71(11 \%)$ \\
Centro académico & $51 / 71(72 \%)$ \\
Centro no académico & $20 / 71(28 \%)$ \\
\hline Años tratando FC en el AM & 7 años (1-40) \\
n casos tratados/año & $44(6-200)$ \\
\hline
\end{tabular}

\section{Preferencias de manejo}

En el caso de fracturas de cuello femoral no desplazadas, sin angulación posterior, la fijación interna y osteosíntesis fue el tratamiento más frecuentemente sugerido (65\%; 46/71). El 58\% (41/71) eligió la fijación con tornillos canulados y 7\% (5/71) la fijación con un tornillo deslizante tipo DHS (Dynamic Hip Screw, Synthes). Por otra parte, el 30\% (21/71) indicó que su tratamiento de elección para la mayoría de las fracturas de cuello femoral no desplazadas en el AM sería una artroplastía.

En el caso de fracturas desplazadas, en pacientes con buen estado funcional (representado como un $\mathrm{AM}$ menor de 80 años, independiente, con adecuado estado cognitivo y movilidad previa) ${ }^{10}$ 94\% (67/71) seleccionó una artroplastía total de cadera (ATC) como el tratamiento quirúrgico de elección y el 4\% una hemiartroplastía (3/71). En pacientes con mal estado funcional (representado como un AM de más de 80 años, dependiente, con una demencia leve y baja movilidad) el $86 \%$ (61/71) seleccionó una hemiartroplastía como tratamiento de elección, 8\% (6/71) una ATC y $6 \%$ otro tratamiento $(4 / 71)$.

En el caso de realizar una ATC, el tipo más frecuentemente realizado fue una ATC híbrida (componente femoral cementado, acetabular no cementado; $48 \%, 34 / 71$ ) y luego una no cementada $(46 \% ; 33 / 71)$. El abordaje más frecuentemente utilizado para realizarla es el abordaje posterior a la cadera en $41 \%$ de las respuestas (29/71) y luego el

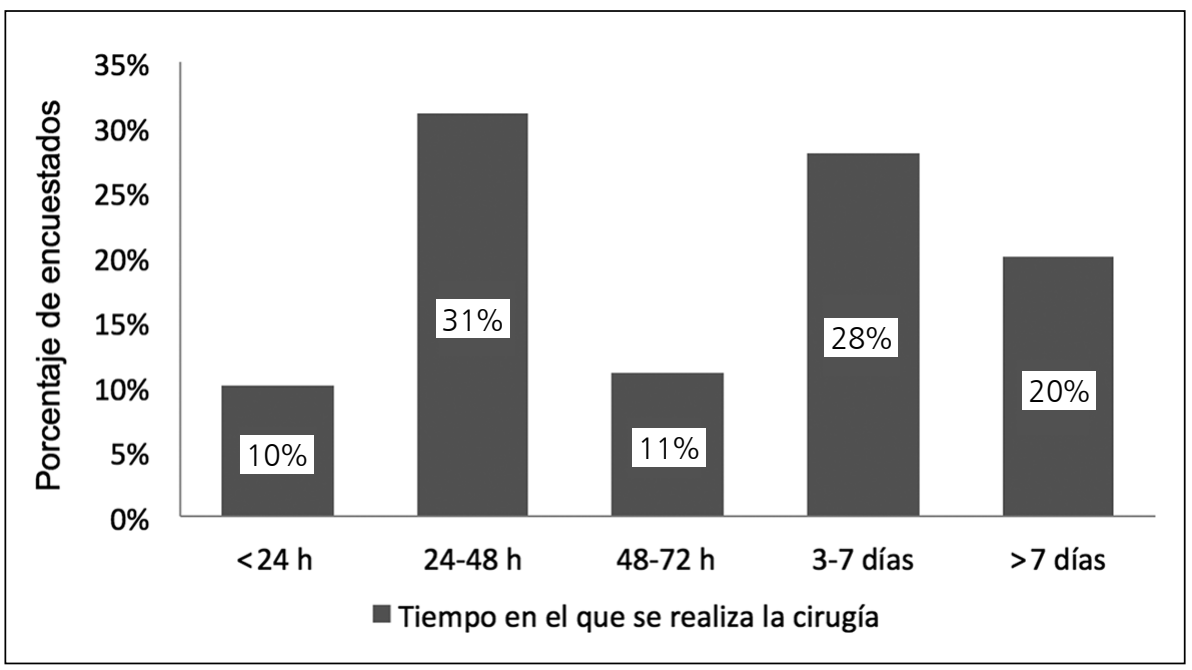

Figura 1. Tiempo aproximado en el que se realiza el tratamiento para un adulto mayor con fractura de cadera. 
Tabla 2. Manejo según tipo de establecimiento donde se desempeña el encuestado

\begin{tabular}{|lcrr|}
\hline & Sector público & $\begin{array}{c}\text { Sector privado } \\
\text { (o mutual/FFAA) }\end{array}$ & p \\
Manejo preoperatorio junto a otra especialidad & $24(68,6 \%)$ & $28(77,8 \%)$ & 0,381 \\
Demora en cirugía $>72 \mathrm{~h}$ & $29(82,9 \%)$ & $5(13,9 \%)$ & $<0,001$ \\
Tratamiento de osteoporosis al alta & $16(45,7 \%)$ & $20(55,6 \%)$ & 0,407 \\
Medición de vitamina D & $2(5,7 \%)$ & $15(41,7 \%)$ & $\mathbf{p}$ \\
& No académico & Académico & 0,001 \\
Manejo preoperatorio junto a otra especialidad & $15(75 \%)$ & $37(72,5 \%)$ & 0,452 \\
Demora en cirugía $>$ 72 h & $11(55 \%)$ & $23(45,1 \%)$ & $<0,001$ \\
Tratamiento de osteoporosis al alta & $4(20 \%)$ & $32(62,7 \%)$ & 0,003 \\
Medición de vitamina D & 0 & $17(33,3 \%)$ & \\
\hline
\end{tabular}

abordaje lateral $(38 \% ; 27 / 71)$, anterolateral $(20 \%$; $14 / 71)$ y anterior $(1 \% ; 1 / 71)$.

En fracturas pertrocantéricas, la gran mayoría de los encuestados eligió realizar una fijación interna y osteosíntesis, 75\% (53/71) utilizando un clavo cefalomedular en la mayoría de sus casos, mientras que 23\% (16/71) utiliza principalmente un dispositivo con tornillo deslizante (DHS). En fracturas subtrocantéricas (definidas como aquellas que involucran los $5 \mathrm{~cm}$ inferiores al trocánter menor), 99\% utiliza un clavo cefalomedular como primera alternativa $(70 / 71)$. En el caso de patrones específicas de fracturas, en fracturas pertrocantéricas simples (AO 31-A1) el tornillo deslizante fue la alternativa utilizada por $68 \%(48 / 71)$, mientras que $31 \%(22 / 71)$ utilizaron un clavo cefalomedular. En fracturas multifragmentarias(AO A2.2) 93\% (66/71) utilizaron un clavo cefalomedular de distinto largo y sólo 7\% (5/71 un tornillo deslizante tipo DHS. En fracturas complejas tipo AO A3.3 97\% (69/71) eligió utilizar un clavo cefalomedular, la mayoría de mayor longitud.

Con respecto al manejo postoperatorio, 51\% de los encuestados refirió realizar de forma regular tratamiento para la osteoporosis (36/71). Además de esto, sólo 39\% (28/71) refirió que iniciaban tratamiento con algún bifosfonato como antiresortivo posterior a esto y $51 \%$ indica alguna combinación de calcio/vitamina D. De forma similar, solo 24\% (17/71), mide niveles plasmáticos de vitamina $\mathrm{D}$ para detectar deficiencia de esta. El 100\% realiza quimio profilaxis para trombosis venosa profunda por un plazo variable entre 1035 días, principalmente con heparinas de bajo peso molecular (HBPM) o alguno de los nuevos anticoagulantes orales (NACO).

Al comparar según el lugar de trabajo, los encuestados que se desempeñaban principalmente en el sector público declararon un mayor tiempo hasta la cirugía $(82,9 \%$ más de $72 \mathrm{~h}$, mientras que solo $13,9 \%$ en el sector privado; $\mathrm{p}<0,001)$ y una menor tasa de medición de niveles plasmáticos de vitamina $D$ en sus pacientes $(5,7 \%$ vs $41,7 \%$ en el sector privado; $\mathrm{p}<0,001)$. De la misma forma, los encuestados que se desempeñaban en un ambiente no académico, refirieron una menor tasa de tratamiento de osteoporosis al alta (20\% vs $62,7 \% ; \mathrm{p}<0,001)$, y una menor tasa de medición de niveles plasmáticos de vitamina $\mathrm{D}$ ( 0 vs $33,3 \%$; $\mathrm{p}=0,003)($ Tabla 2).

\section{Discusión}

La fractura de cadera en el adulto mayor es una situación clínica frecuente y grave. Es así, como ante la creciente evidencia que apoya distintas prácticas en su manejo, es fundamental realizar un diagnóstico adecuado de como estamos actuando a nivel nacional $y$, de esta forma, poder intervenir para mejorar los resultados de nuestros pacientes.

Los resultados de esta encuesta muestran varios elementos claves: 1) La gran mayoría de los encuestados esta de acuerdo con el manejo preoperatorio multidisciplinario y su impacto en el resultado en estos pacientes. 2) A pesar de que la mayoría está de acuerdo en que el manejo quirúrgico en menos de $72 \mathrm{~h}$ es fundamental, esto 
se realiza en tan sólo la mitad de las prácticas, con claras diferencias en acceso si el sistema es público o privado. 3) El manejo quirúrgico general es constante para la mayoría de los escenarios. Fracturas desplazadas de cuello femoral son manejadas con una artroplastía total o parcial según estado funcional y fracturas per o subtrocantéricas con una reducción y fijación interna. La artroplastía para la fractura de cuello femoral no desplazada aparece como una alternativa importante $(30 \%$ de las respuestas), en línea con controversias internacionales ${ }^{1,11}$.4) Sólo la mitad de los cirujanos declara realizar un manejo metabólico y hacerse cargo de la prevención secundaria de osteoporosis, con diferencias según prácticas académicas o no académicas.

Este estudio tiene limitaciones y fortalezas. Por una parte, sus resultados deben ser interpretados en el contexto de su metodología. Una encuesta no necesariamente representa el manejo específico de pacientes con cierta patología, sino más bien la opinión general sobre un escenario clínico, con sesgos de memoria y de juicio que pueden alterar los resultados. Por otra parte, solo se encuestó un fragmento pequeño de los traumatólogos a nivel nacional, probablemente sobre representando la Región Metropolitana, el ambiente académico y médicos subespecializados. En contraste, las fortalezas de nuestro estudio incluyen el uso de un proceso riguroso para desarrollar el cuestionario, con un piloteo adecuado y una muy buena tasa de respuesta. Además de esto, la encuesta fue basada en una amplia revisión de la literatura, temas contingentes y la participaciones de cirujanos de amplia experiencia.

El manejo preoperatorio en la FC en el AM ha sido ampliamente revisado ${ }^{1,12}$. Una cirugía precoz se ha asociado a menor mortalidad temprana y tardía, así como menor tiempo de estadía hospitalaria, mejor manejo del dolor y retorno a la vida independiente ${ }^{13-16}$. La recomendación más ampliamente utilizada es menos de 48-72 h, pero con tendencia a considerar tiempos aún menores. Casi la mitad de los encuestados refirieron que en sus centros el tiempo hasta la cirugía es por lo general mayor a 3 días y un quinto de ellos que es mayor a 7 días, cifras que aumentan de forma importante si consideramos solamente aquellos encuestados que se desempeñan en el sector público. Existen escasos reportes nacionales, sin embargo, las cifras distan ampliamente de las recomendaciones internacionales, con tiempos de demora hasta la cirugía de 19 días promedio y sólo $7 \%$ de los pacientes siendo operados antes de los primeros 5 días?.

Clásicamente los distintos tipos de FC en el AM han sido tratados según el lugar anatómico comprometido en el fémur proximal, el desplazamiento y la funcionalidad del paciente. En el caso de fracturas de cuello femoral, amplia evidencia soporta la resolución quirúrgica con una artroplastia en caso de fracturas desplazadas ${ }^{15}$, con menores tiempos de rehabilitación y riesgo de complicaciones que otras alternativas. En el caso de fracturas de cuello femoral no desplazadas, el tratamiento más frecuentemente utilizado es la fijación interna con el uso de tornillos canulados, al igual a lo observado en nuestra encuesta. El uso de una artroplastía en el caso de fracturas no desplazadas de cuello femoral en el AM es un tema controversial. Reportes que muestran menores tasas de reoperación y mejores resultados funcionales ${ }^{17,18}$ hacen que su uso sea una alternativa atractiva, como lo representa la respuesta de $30 \%$ de los encuestados. Sin embargo, un mayor riesgo global y complicaciones más graves, hacen necesaria una mejor evidencia sobre este tema ${ }^{19,20}$ antes de poder generalizar una recomendación. En el caso de fracturas que comprometen la región per y subtrocantéricas, el tratamiento principal es la reducción y fijación interna, independiente del desplazamiento.

En cuanto al manejo de la osteoporosis, la mitad de los traumatólogos encuestados refiere indicar de forma regular algún tipo de tratamiento, principalmente combinaciones de calcio y vitamina $\mathrm{D}$, mientras que sólo $39 \%$ refirió utilizar un antiresortivo de forma habitual en el manejo de estos pacientes. Reportes previos muestran una baja tasa de tratamiento para osteoporosis posterior a una fractura de cadera, tanto a nivel nacional ${ }^{21}$ como internacional $^{22,23}$, con cifras entre 0 y $28,5 \%$. Las razones detrás de la diferencia entre las recomendaciones de tratamiento basadas en la evidencia y la práctica habitual pueden ser variadas y no han sido claramente estudiadas. De la misma forma, la medición de niveles plasmáticos de Vitamina $\mathrm{D}$ y por ende su repleción y suplementación posterior, se realiza en niveles menores, a pesar de evidencia que muestra un alta proporción de insuficiencia en adultos mayores con fractura de cadera en nuestra población ${ }^{24}$. 
El manejo de AM con una FC es complejo y tiene muchas aristas. A pesar de la amplia evidencia disponible, existen diferencias en las prácticas entre distintos especialistas que no pueden ser reducidas a una simple causa. Diferencias en manejo pueden deberse a desconocimiento de la mejor evidencia, falta de acceso a cierto tratamiento, que no existe superioridad demostrada entre un enfrentamiento y otro, desacuerdos en la interpretación de la información científica, en experiencia, valores e incluso preferencias personales.

En nuestro país se hacen claras las diferencias en el acceso a la salud al momento de enfrentar una situación como la FC en el AM, y las capacidades que tienen los equipos clínicos para resolver este escenario de forma precoz y efectiva. Por otra parte, la falta de adherencia a guías establecidas en el manejo postoperatorio de pacientes con un claro diagnóstico de osteoporosis hacen sospechar una falta de rigurosidad al momento de tratar a un paciente de forma global, pero también puede reflejar una falta de colaboración entre especialistas o sobrecarga asistencial. Futuras líneas de investigación deberían profundizar en este diagnóstico, para así poder gestionar las intervenciones necesarias para asegurar un mejor resultados de nuestros pacientes.

\section{Referencias}

1. Zamora T, Klaber I, Urrutia J. Hip Fracture in the Elderly. Clinical Medicine Insights: Geriatrics 2017; 10: 1-10.

2. Mears SC, Kates SL. A Guide to Improving the Care of Patients with Fragility Fractures, Edition 2. Geriatr Orthop Surg Rehabil 2015; 6 (2): 58-120.

3. Osnes EK, Lofthus CM, Meyer HE, Falch JA, Nordsletten L, Cappelen I, et al. Consequences of hip fracture on activities of daily life and residential needs. Osteoporos Int 2004; 15 (7): 567-74.

4. Becker DJ, Kilgore ML, Morrisey MA. The societal burden of osteoporosis. Curr Rheumatol Rep 2010; 12 (3): 186-91.

5. Brauer CA, Coca-Perraillon M, Cutler DM, Rosen AB. Incidence and mortality of hip fractures in the United States. JAMA 2009; 302 (14): 1573-9.

6. Amin S, Achenbach SJ, Atkinson EJ, Khosla S, Melton LJ, 3rd. Trends in fracture incidence: a population-based study over 20 years. J Bone Miner Res 2014; 29 (3): 5819.

7. Braithwaite RS, Col NF, Wong JB. Estimating hip frac- ture morbidity, mortality and costs. J Am Geriatr Soc 2003; 51 (3): 364-70.

8. Vásquez I, Valenzuela C, Zapata C, Ruz S, Verones V, Iglesias V, et al. Prevalencia de fractura de cadera en adulto mayores institucionalizados en Ia Provincia Santiago. Rev Chil Salud Pública 2005; 9 (1): 39-45.

9. Dinamarca-Montecinos JL, Amestica-Lazcano G, Rubio-Herrera R, Carrasco-Buvinic A, Vásquez A. [Hip fracture. Experience in 647 Chilean patients aged 60 years or more]. Rev Med Chile 2015; 143 (12): 15529.

10. Rogmark C, Carlsson A, Johnell O, Sernbo I. A prospective randomised trial of internal fixation versus arthroplasty for displaced fractures of the neck of the femur. Functional outcome for 450 patients at two years. J Bone Joint Surg Br 2002; 84 (2): 183-8.

11. Palm H, Gosvig K, Krasheninnikoff M, Jacobsen S, Gebuhr P. A new measurement for posterior tilt predicts reoperation in undisplaced femoral neck fractures 113 consecutive patients treated by internal fixation and followed for 1 year. Acta Orthopaedica 2009; 80 (3): 303-7.

12. Bengoa F, Carrasco M, Amenábar Edwards PP, Schweitzer D, Botello Correa E, Klaber I. Optimización perioperatoria del paciente anciano con fractura osteoporótica de cadera 2017.

13. Grimes JP, Gregory PM, Noveck H, Butler MS, Carson JL. The effects of time-to-surgery on mortality and morbidity in patients following hip fracture. Am J Med 2002; 112 (9): 702-9.

14. Orosz GM, Magaziner J, Hannan EL, Morrison RS, Koval K, Gilbert M, et al. Association of timing of surgery for hip fracture and patient outcomes. JAMA 2004; 291 (14): 1738-43.

15. Roberts KC, Brox WT. AAOS Clinical Practice Guideline: Management of Hip Fractures in the Elderly. J Am Acad Orthop Surg 2015; 23 (2): 138-40.

16. Moja L, Piatti A, Pecoraro V, Ricci C, Virgili G, Salanti $\mathrm{G}$, et al. Timing matters in hip fracture surgery: patients operated within 48 hours have better outcomes. A meta-analysis and meta-regression of over 190,000 patients. PloS one 2012; 7 (10): e46175.

17. Gjertsen JE, Engesaeter LB, Furnes O, Havelin LI, Steindal K, Vinje T, et al. The Norwegian Hip Fracture Register: experiences after the first 2 years and 15,576 reported operations. Acta Orthop 2008; 79 (5): 583-93.

18. Gjertsen JE, Fevang JM, Matre K, Vinje T, Engesaeter LB. Clinical outcome after undisplaced femoral neck fractures. Acta Orthop 2011; 82 (3): 268-74.

19. Griffin J, Anthony TL, Murphy DK, Brennan KL, Brennan ML. What is the impact of age on reoperation rates 
for femoral neck fractures treated with internal fixation and hemiarthroplasty? A comparison of hip fracture outcomes in the very elderly population. J Orthop 2016; 13 (1): 33-9.

20. Lin JC, Liang WM. Outcomes after fixation for undisplaced femoral neck fracture compared to hemiarthroplasty for displaced femoral neck fracture among the elderly. BMC Musculoskelet Disord 2015; 16: 199.

21. Rodríguez PJA, Borzutzky S A, Barnett T C, Marín L PP. Falta de reconocimiento de osteoporosis y omisión de tratamiento en adultos mayores con fractura de cadera en Chile. Rev Med Chile 2003; 131: 773-8.
22. Rabenda V, Vanoverloop J, Fabri V, Mertens R, Sumkay $\mathrm{F}$, Vannecke C, et al. Low incidence of anti-osteoporosis treatment after hip fracture. J Bone Joint Surg Am 2008; 90 (10): 2142-8.

23. Solomon DH, Johnston SS, Boytsov NN, McMorrow D, Lane JM, Krohn KD. Osteoporosis medication use after hip fracture in U.S. patients between 2002 and 2011. J Bone Miner Res 2014; 29 (9): 1929-37.

24. Schweitzer D, Amenábar PP, Botello E, López M, Saavedra Y, Klaber I. Prevalencia de insuficiencia y deficiencia de vitamina $\mathrm{D}$ en adultos mayores con fractura de cadera en Chile. Rev Med Chile 2016; 144: 175-80. 